\title{
Unveiling nonlinear regimes of light amplification in fused silica with femtosecond imaging spectroscopy
}

\author{
Thomas Winkler $\odot^{*}$ \\ Institute of Physics and CINSaT, University of Kassel, Heinrich-Plett-Str. 40, 34132 Kassel, Germany \\ and Department of Physics and Astronomy, Aarhus University, 8000 Aarhus C, Denmark \\ Peter Balling $($ ) \\ Department of Physics and Astronomy, Aarhus University, 8000 Aarhus C, Denmark
}

\begin{abstract}
Bastian Zielinski $\odot$, Cristian Sarpe, Nikolai Jelzow, Ramela Ciobotea $\odot$, Arne Senftleben, and Thomas Baumert Institute of Physics and CINSaT, University of Kassel, Heinrich-Plett-Str. 40, 34132 Kassel, Germany
\end{abstract}

(Received 10 November 2019; revised manuscript received 16 December 2019; accepted 27 May 2020;

published 15 June 2020)

\begin{abstract}
We observe and characterize two nonlinear light-amplification regimes with unique spectral properties and fluence dependencies in laser-excited fused silica using ultrafast pump-probe imaging spectroscopy. The two underlying mechanisms are switchable within $150 \mathrm{fs}$ via the time delay between an intense near-infrared femtosecond pump and an ultraviolet femtosecond probe pulse. While the earlier amplification regime is linked to the effect of laser amplification in excited dielectrics, and thus to the presence of conduction-band electrons, the latter can be directly related to the ultrafast formation of extremely long-lived self-trapped excitons. It is shown that, locally, the amplification can reach one order of magnitude. We discuss the temporal and spectral characteristics of the amplification as well as its dependencies on pump and probe fluences and form a complete picture within a tentative model.
\end{abstract}

DOI: 10.1103/PhysRevResearch.2.023341

\section{INTRODUCTION}

The coherent amplification of light in doped glasses or semiconductors is well known. However, in recent years, a variety of previously unknown light-amplification mechanisms in high-bandgap materials have been revealed, of which amplification by nearly free electrons in gases [1] and by laser-induced Kerr instabilities in dielectrics [2] are only two of many thrilling examples. Although based on different mechanisms, the amplification is linear in both cases. In contrast, laser amplification in excited dielectrics (LADIE) is a recent discovery, in which an ultrashort 400-nm femtosecond laser pulse is nonlinearly amplified inside an optically excited dielectric sample via multiphoton stimulated emission [3]. As the origin of LADIE is dependent on the presence of conduction-band (CB) electrons and valence-band (VB) holes, its lifetime is restricted by the time scale of carrier recombination [3]. This was evidenced in the case of excited fused silica, where the ultrafast formation of self-trapped excitons (STEs) diminished the conditions for the LADIE

\footnotetext{
*winkler@phys.au.dk

Published by the American Physical Society under the terms of the Creative Commons Attribution 4.0 International license. Further distribution of this work must maintain attribution to the author(s) and the published article's title, journal citation, and DOI.
}

effect on the ultrashort time scale of 100 to $200 \mathrm{fs}$ [3-5]. In this work, we report the surprising observation of a second, temporally separated nonlinear amplification process, which is directly linked to the presence of the long-lived STE states and thus termed light amplification with self-trapped exciton resonances (LASTER). We present a detailed characterization of the amplification of an ultrashort 400-nm pulse in both regimes, LADIE and LASTER, and relate the results to a proposed mechanism. The measurements include the investigation of the dependencies on the laser-pulse fluence, as well as the temporal and spectral characteristics of the amplifications, in order to highlight similarities and differences between the regimes, with emphasis on the newly discovered STE-based amplification.

Optical excitation of dielectric materials, such as sapphire, fused silica, and water, can induce a variety of effects in materials. Transient effects from white-light and high-harmonics generation [6] to permanent refractive index changes [7-9] or even material ablation [10-12] are prominent examples. In recent years, in situ investigations utilizing ultrafast pumpprobe and spectral-interferometry techniques were used to shine light on the interaction of femtosecond laser pulses with high-bandgap dielectrics [3,10,13-15]. Understanding the fundamental processes is of great importance also because femtosecond laser pulses have become a tool of choice for high-precision material processing on the micro- and nanometer scale in solid and liquid dielectrics [16-19]. In this work, we focus on the transient optical changes in a thin fused-silica 

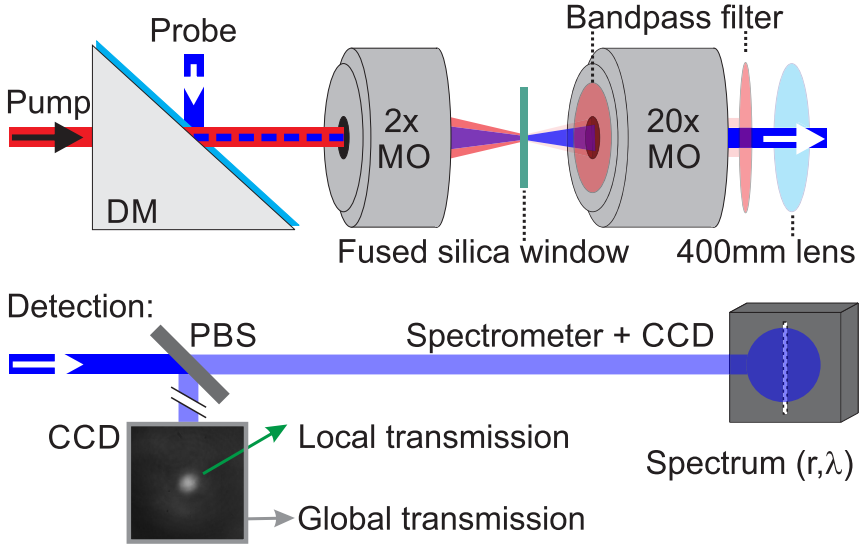

FIG. 1. Sketch of the femtosecond imaging pump-probe setup. We utilize single, 800-nm (29-fs-FWHM) pump pulses for the excitation, whereas time-delayed single 400-nm (45-fs-FWHM) pulses are used to probe the material changes of the $100-\mu$ m-thin fused silica window. Pump and probe beams are combined using a dichroic mirror (DM), while a $2 \times$ apochromatic infinity-corrected microscope objective (MO) is used to focus both pulses together onto the sample. A $20 \times$ apochromatic infinity-corrected MO is used to collect the probe pulse and a 400-mm-focal-length lens images the surface of the sample onto a monochromatic CCD camera and an imaging spectrometer. The use of a pellicle beam splitter (PBS) avoids the creation of ghost images. The remaining pump light is removed by two bandpass filters. The CCD camera allows us to extract the local and global (spatially integrated) signal; the spectrometer is used to obtain the spatially resolved spectrum $[S(r, \lambda)]$ of the probe pulse. With measured $1 / e^{2}$ beam waists of 12 and $28 \mu \mathrm{m}$ for the pump and probe beam, their Rayleigh ranges exceed the sample thickness by at least five times $(0.56$ and $6.16 \mathrm{~mm})$-allowing homogeneous illumination of the sample. The full experimental setup is described in the methods section.

sample following excitation by a short, 29-fs-FWHM, 800-nm pump pulse, measured with a femtosecond imaging setup as sketched in Fig. 1.

\section{TEMPORAL DYNAMICS}

We initially discuss the transmission of the ultrashort 400$\mathrm{nm}$ probe pulse as a function of the delay time after the 800-nm pump pulse. CCD images of the transmitted probe pulse at five delay times (marked on the delay-time axis) are shown in Fig. 2(a). As expected, prior to excitation with the pump pulse [Fig. 2(a), i], the probe pulse shows a Gaussian fluence distribution. The transmitted fluence distribution drastically changes when the pump and probe pulses overlap in time [Fig. 2(a), ii]. Now the probe pulse undergoes a strong spatial redistribution towards much larger radii [Fig. 2(b)] combined with an increasing global transmission [black circles in Fig. 2(c)], where the spatially resolved transmission in Fig. 2(b) is obtained from a radial cut through the CCD data. The data for the center of the pulse are reproduced in Fig. 2(c) as local transmission (green squares), while "global" transmission refers to the spatially integrated signal. The changes observed at zero time delay are based on the optical Kerr effect (OKE) [20], which causes a transient intensity-dependent refractive-index change induced by the
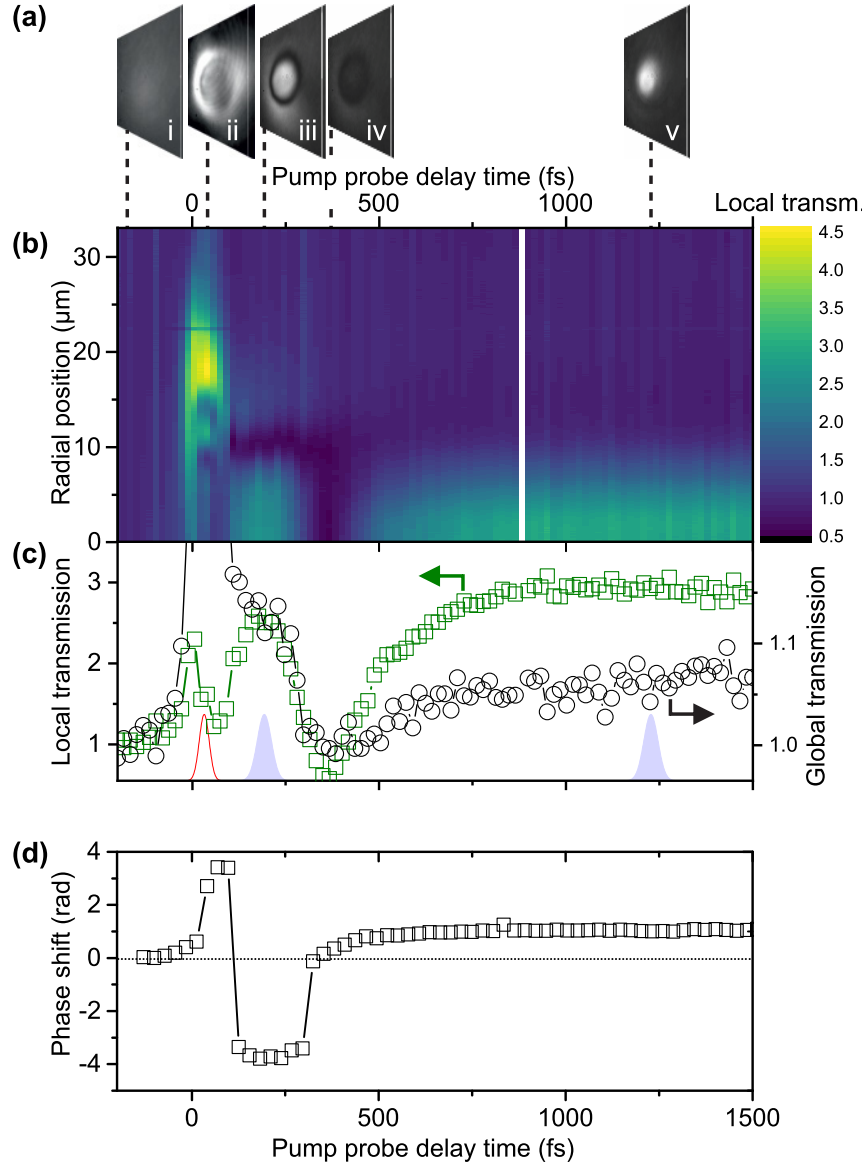

FIG. 2. Temporal dynamics of laser-excited fused silica, probed with a 400-nm probe pulse, using a pump-pulse peak fluence of 2 $\mathrm{J} \mathrm{cm}^{-2}$ and a probe-pulse peak fluence of $33 \mathrm{~mJ} \mathrm{~cm}^{-2}$. (a) CCD images of the probe pulse after passing the fused silica sample at a pump-probe delay time of (i) $200 \mathrm{fs}$, (ii) $0 \mathrm{fs}$, (iii) $200 \mathrm{fs}$, (iv) $375 \mathrm{fs}$, and (v) $1200 \mathrm{fs}$. CCD images show a magnified $58.5 \times 58.5 \mu \mathrm{m}$ region of the sample and are put in perspective for visualization purposes. (b) Radially resolved local transmission of the probe pulse as a function of the pump-probe delay time. (c) Local transmission extracted from the center of the probe pulse (green squares) and global transmission (black circles). The Gaussian functions in (c) represent the temporal profiles of the pump (red line) and probe (blue-shaded areas) pulses, shown at delay times iii and iv. (d) Phase-shift measurement obtained from the center of the probe pulse in a separate spectral-interference measurement performed under identical pump conditions.

pump pulse, resulting in cross-phase modulation and other coupling mechanisms between the two pulses [2,21]. As soon as the pump and probe pulses are clearly separated in time (after around $100 \mathrm{fs}$ ), we observe a strong local amplification up to a factor of 2.5 in the center of the probe pulse (from 0 to $10 \mu \mathrm{m}$ in radii) and absorption at larger radii (10 to $15 \mu \mathrm{m})$. The global transmission reaches a value of 1.15 , and at the same time, under identical excitation conditions, the highly negative phase shift of $-4 \mathrm{rad}$ can be measured [Fig. 2(d)], indicating the presence of a high electron density in the conduction band $[3,10,13,22]$. An increasing delay leads to a reduction of the transmission with a minimum at around $350 \mathrm{fs}$, where absorption is observed in the center of 
(a)

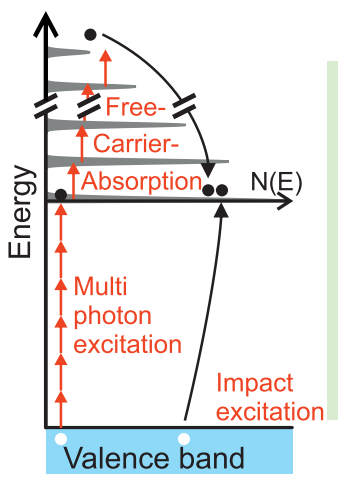

(b)

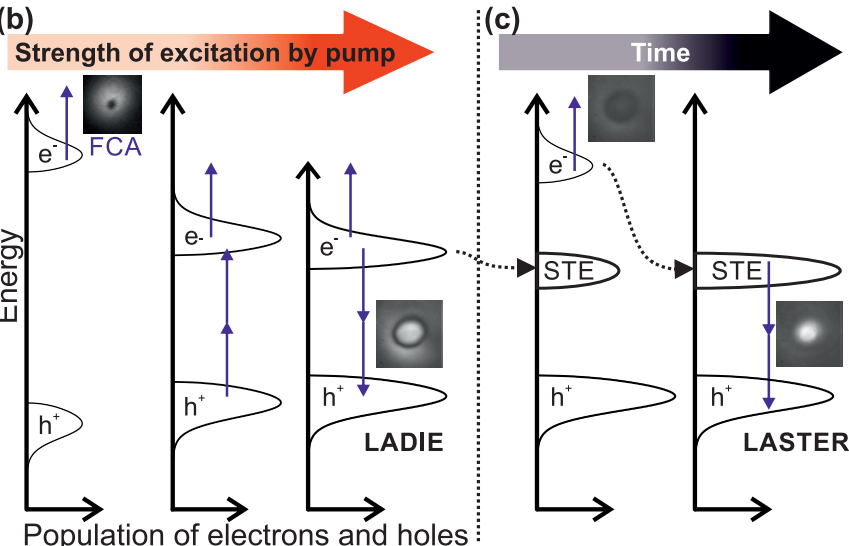

FIG. 3. Sketch of the proposed amplification mechanisms. (a) Sketch of the multiphoton and impact excitation of valence-band electrons into the conduction band by the intense 800-nm pump pulse followed by intraband thermalization. (b) Sketch of the influence of excitation strength. Left: Free-carrier absorption of the probe pulse by conduction-band electrons. Middle: Bandgap renormalization allows two-photon absorption. Right: At higher excitation strengths, an energetically localized population inversion allows two-photon stimulated emission. (c) The ultrafast formation of self-trapped excitons causes a drastic reduction of the conduction-band electron density leading to the fading of the LADIE effect (left), followed by the formation of an energetically localized population inversion (right) between the self-trapped excitons and the top of the valence band, giving rise to a second nonlinear stimulated emission process-LASTER.

the probe pulse [Fig. 2(a), iv]. At the same time, the phase shift measurement [Fig. 2(d)] shows a transition from a negative to a positive value, which is the characteristic optical signature of the ultrafast creation of long-lived STEs $[5,10,23,24]$. When the pump-probe delay time is increased to larger values, absorption vanishes and is replaced by amplification, leading to a local transmission coefficient close to 3 [Fig. 2(c); green squares] and a global transmission of 1.05 to 1.075 (black circles). The maximum local transmission is reached within $800 \mathrm{fs}$ and stays constant over more than $1 \mathrm{~ns}$, which is the limit given by our experimental setup. Compared to the first amplification regime at 100 to $300 \mathrm{fs}$, the local transmission is higher but more confined ( 0 to $5 \mu \mathrm{m}$ ). However, we observe that the global transmission is lower. This can be understood by the high amplification region being confined to smaller radii, hence affecting a smaller area of the probe pulse such that the overall change is lower. In this study, we focus on the two regimes of amplification that are present after the excitation by the pump pulse [time steps iii and $\mathrm{v}$ in Fig. 2(a)]. Therefore, first, we discuss the possible mechanisms that lead to the appearance of the two amplification regimes. Next, we discuss experimental results that demonstrate the differences and similarities between the amplification regimes and how the results corroborate the proposed model.

\section{PROPOSED MECHANISM}

The sample is a low-defect-density, amorphous, $100-\mu \mathrm{m}$ thin and polished UV-grade fused silica window with a bandgap energy of more than $9 \mathrm{eV}$. The intense $800-\mathrm{nm}$, 29 -fs pump pulse will initially excite electrons from the valence band to the conduction band via strong-field (e.g., multiphoton) excitation, involving at least six photons $[11,25]$. Due to ponderomotive energy shifts [11,25], the bandgap energy will increase dynamically with the intensity, leading to even higher-order multiphoton-ionization/excitation (MPI) processes. Once electrons are present in the $\mathrm{CB}$, they can gain energy via free-carrier absorption (FCA), as indicated in Fig. 3(a). As the CB electrons reach sufficiently high energies, they can collisionally excite another electron from the VB. Subsequently, the process starts again, eventually leading to an avalanchelike multiplication of the carrier density. After the pump pulse passes the sample, the initially steplike energy distribution of electrons and holes [see the sketch in Fig. 3(a)] will be redistributed via intraband thermalization towards a quasi-Fermi distribution by intraband scattering processes on the short time scale of less than $100 \mathrm{fs}[3,26]$. At this time, the delayed probe pulse exhibits FCA by the CB electrons, as sketched on the left in Fig. 3(b). While this (linear) intraband FCA is always present as long as free carriers exist, a nonlinear interaction [e.g., a two-photon absorption (TPA) from $\mathrm{VB}$ to $\mathrm{CB}$ ] of the probe pulse is highly dependent on its intensity. In the unexcited state of fused silica, at least three probe-pulse photons are required to overcome the bandgap energy of $9 \mathrm{eV}$. Consequently, without pumping the sample (with the 800-nm pulse), we do not observe a measurable change in the probe-pulse transmission when the fused silica sample is inserted into the beam path, besides interfacial reflection. This shows that the probe pulse does not have sufficient intensity to drive a measurable three-photon absorption. However, as soon as the sample is excited by the pump pulse, many-body interactions of the carriers will lead to a carrier-density-dependent bandgap renormalization [27-29]. This renormalization can reach large values in semiconductors [30-32] and high-bandgap dielectrics, as shown theoretically for diamond [28]. If the bandgap renormalization (and probepulse intensity) is sufficiently strong, nonlinear TPA of the probe pulse as sketched in Fig. 3(b) (middle) becomes possible. As the carrier density increases (i.e., at higher pump fluences), the effects of bandgap renormalization are stronger [28], and at the same time, the density of carriers close to the band edges (due to the quasi-Fermi distribution) increases significantly, leading to a decrease in TPA due to ground-state bleaching/Pauli blocking. Moreover, the increase in carrier 
density at the edges of the bandgap will eventually lead to a situation in which more electrons are located at the bottom of the CB than at the top of the VB. To be specific, an energetically localized population inversion is created at or close to the transition energy of the two-photon process (approx $6 \mathrm{eV}$ ). The immediate consequence is that the TPA turns into degenerate two-photon stimulated emission, meaning that two incident photons (of the same wavelength) lead to the emission of two additional ones, causing amplification of the probe pulse. As noted earlier, the observation of the nonlinear amplification process that is correlated with the presence of conduction-band electrons has been previously observed in a 100- $\mu \mathrm{m}$-thin sapphire window and labeled LADIE [3]. In this study, we also modeled the LADIE process using a multiple-rate equation model $[33,34]$ for the excitation, followed by intraband thermalization and one-dimensionalpropagation calculations, obtaining excellent qualitative and semiquantitative agreement between the modeling and the experimental results [3]. We showed that an excitation of only $0.5 \%$ of the $\mathrm{VB}$ electrons (i.e., a density of $\sim 10^{21} \mathrm{~cm}^{-3}$ ) is sufficient to reach the threshold for an energetically localized population inversion. As the amplification conditions are strongly linked to a certain carrier density, the amplification only takes place in a thin layer of the material, close to the surface. This is caused by the strong absorption of the pump pulse due to MPI and FCA, leading to an intensity, and thus a carrier density, that drastically decreases within the first hundreds of nanometers [3]. Therefore, the probe pulse encounters nonlinear amplification, as well as nonlinear and free-carrier absorption in different regions on its way through the sample, limiting the overall amount of amplification. With similar bandgap energies and material properties [10], the modeling results obtained on sapphire can be directly applied to the fused silica shortly after the excitation. This assumption is supported by the observation of similar spectral properties of the amplification and nearly identical probe- and pump-pulse fluence dependencies, as discussed in the next sections.

If the delay time between pump and probe pulses is increased above approx $250 \mathrm{fs}$, the majority of CB electrons are captured into the long-lived self-trapped exciton states. These states are located in the middle of the bandgap around $5.4 \mathrm{eV}$ above the VB edge [23,24] and have characteristic lifetimes of the order of several microseconds [35]. During this process, the CB electron density is rapidly decreasing, and the conditions for LADIE (i.e., population inversion and bandgap renormalization) are abating, causing FCA once again to become the prominent feature in the measurement, as sketched in Fig. 3(c) (left) and identified in the experiment by time step "iii" [Fig. 2(a), iv]. However, with increasing delay time, the majority of CB electrons are trapped in STE states, leading again to an energetically localized population inversion, this time between the STE states and the top of the VB; this allows a two-photon emission process that can be stimulated by the intense probe pulse, leading to its amplification [Fig. 3(c) (right); time step "v"]. As mentioned above, this amplification is named light amplification with self-trapped exciton resonances. The relaxation of electrons from the $\mathrm{CB}$ into the VB takes place on a much longer time scale and is therefore neglected. In strong contrast to LADIE, where
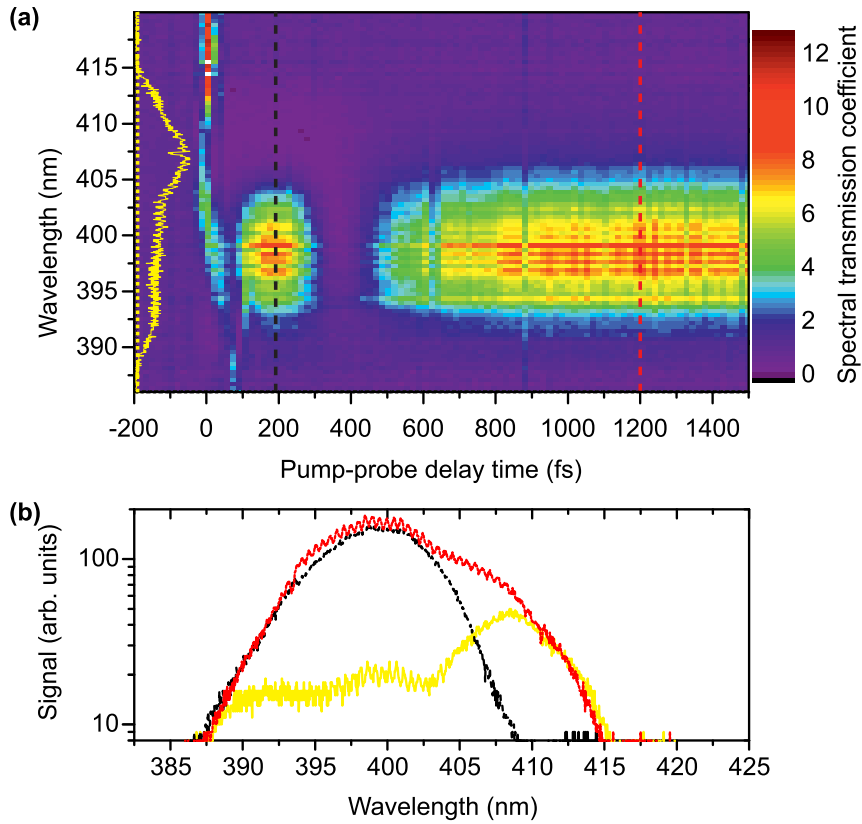

FIG. 4. Spectral properties of the probe pulse as a function of the delay time. (a) Spectral transmission coefficient as a function of the pump-probe delay time, obtained from the spatial center of the probe pulse [which corresponds to the data in Fig. 2(c); green squares]. (b) Logarithmic representation of the measured spectrum of the probe pulse obtained at a time delay of $-200 \mathrm{fs}$ (yellow curve), $200 \mathrm{fs}$ (dotted black curve), and $1200 \mathrm{fs}$ (dashed red curve) using a pump-pulse peak fluence of $2 \mathrm{~J} \mathrm{~cm}^{-2}$. The delay times at which the spectra were taken are indicated by the corresponding vertical lines in (a).

the probe pulse encounters very different processes along its path through the material (amplification, nonlinear and linear absorption), at late time delays, the probe pulse will only propagate through parts of the material which either exhibit amplification or are transparent.

In the following section, we present and discuss differences in the spectral and energetic properties of LADIE and LASTER.

\section{DIFFERENCE BETWEEN THE AMPLIFICATION REGIMES}

\section{A. Spectral properties of LADIE and LASTER}

Let us now consider the spectral properties of the transmitted 400-nm probe pulse: Fig. 4(a) shows the spectrally resolved transmission as a function of the pump-probe delay time, taken at the center of the probe pulse. This scan corresponds to the measurement shown in Fig. 2(d), as the data were recorded simultaneously. For reference, the initial probe-pulse spectrum is shown as the vertical yellow curve in the range of negative delay times in Fig. 4(a). When the delay time approaches 0 , we observe a strong increase in spectral transmission over all parts of the probe spectrum and beyond. This is caused by the cross-phase modulation due to the pump-intensity-dependent optical Kerr effect. At low pump fluences, this signal is used to determine the probe pulse duration $[3,22]$ to be approx $45 \mathrm{fs}$. In the LADIE regime 
(100- to 300-fs delay time), we observe that the spectrum is not amplified homogeneously across all wavelengths. Instead, in the range of 395 to $402 \mathrm{~nm}$, an amplification factor of nearly 10 is achieved, whereas longer wavelengths (407 to $415 \mathrm{~nm})$ are strongly absorbed $(T<0.3)$, showing that the conditions for amplification are only fulfilled in a certain spectral regime, whereas for other wavelengths FCA is dominant. This becomes even more apparent by directly comparing the absolute signal of the reference (yellow line) to the spectrum taken at $200 \mathrm{fs}$ (dotted black line) in Fig. 4(b). The specific amplification and absorption profile leads to a strong shift in the peak of the spectrum from 410 to $400 \mathrm{~nm}$ for the amplified probe pulse. Overall, the measured spectral characteristics are qualitatively identical to those of the LADIE effect observed in sapphire [3]. In the case of LASTER (time delay > $350 \mathrm{fs}$ ), the spectral transmission coefficient [Fig. 4(a)] shows different characteristics compared to the LADIE amplification: We observe a slightly stronger amplification over the larger wavelength range of 390 to $410 \mathrm{~nm}$ and no sign of absorption. For wavelengths above $410 \mathrm{~nm}$, the probe pulse is unaffected, as the measured spectrum is identical to the reference [Fig. 4(b); dashed red line]. This measurement shows that the spectrum under LASTER conditions is broader not only due to the missing FCA, but also due to the broader amplification towards longer wavelengths, which indicates "population inversion" for a wider energy range compared to the band-edge inversion of the LADIE effect. The minor interference pattern, visible in all spectra, is caused by the spectral interference of the probe pulse with its internally reflected copy and can be used to measure the sample thickness [3].

\section{B. Excitation-strength dependence}

As discussed earlier, the conditions for LADIE and LASTER are clearly linked to a certain level of excitation; we thus discuss in the following the dependence on the pumppulse peak fluence. We varied the energy of the pump pulse at two distinct delay times, 200 and $1200 \mathrm{fs}$ [Fig. 2(a); time steps iii and iv], respectively. The pump-fluence scans, shown in Figs. 5(a)-5(c), are limited to fluence values below the measured ablation threshold $\left(2.5 \mathrm{~J} \mathrm{~cm}^{-2}\right)$. Please note that we define "ablation" as the pump-pulse peak fluence at which any permanent material change can be observed with our pump-probe microscope after a single pulse.

In the case of a 200-fs time delay, corresponding to LADIE, we observe that with increasing pump-pulse peak fluence above $0.85 \mathrm{~J} \mathrm{~cm}^{-2}$, the local transmission of the probe pulse in the center [Fig. 5(a) and green squares in Fig. 5(b)] decreases, which can be explained by strong FCA $[3,11]$. However, for pump fluences above $1.25 \mathrm{~J} \mathrm{~cm}^{-2}$, we observe an increase in transmission, reaching its maximum value at $2.25 \mathrm{~J} \mathrm{~cm}^{-2}$ before it decreases again, as shown in Fig. S1 in the Supplemental Material [36]. This pump-fluence dependence is nearly identical to the one measured in excited sapphire [3], showing the similarities between the two materials under these conditions and the generality of the LADIE mechanism. The reason for the decreasing amplification/transmission at higher pump fluences is the ever increasing FCA, combined with the increasing reflection at the surface [3]. From the spatially resolved data shown in Fig. 5(a), it can be seen that the
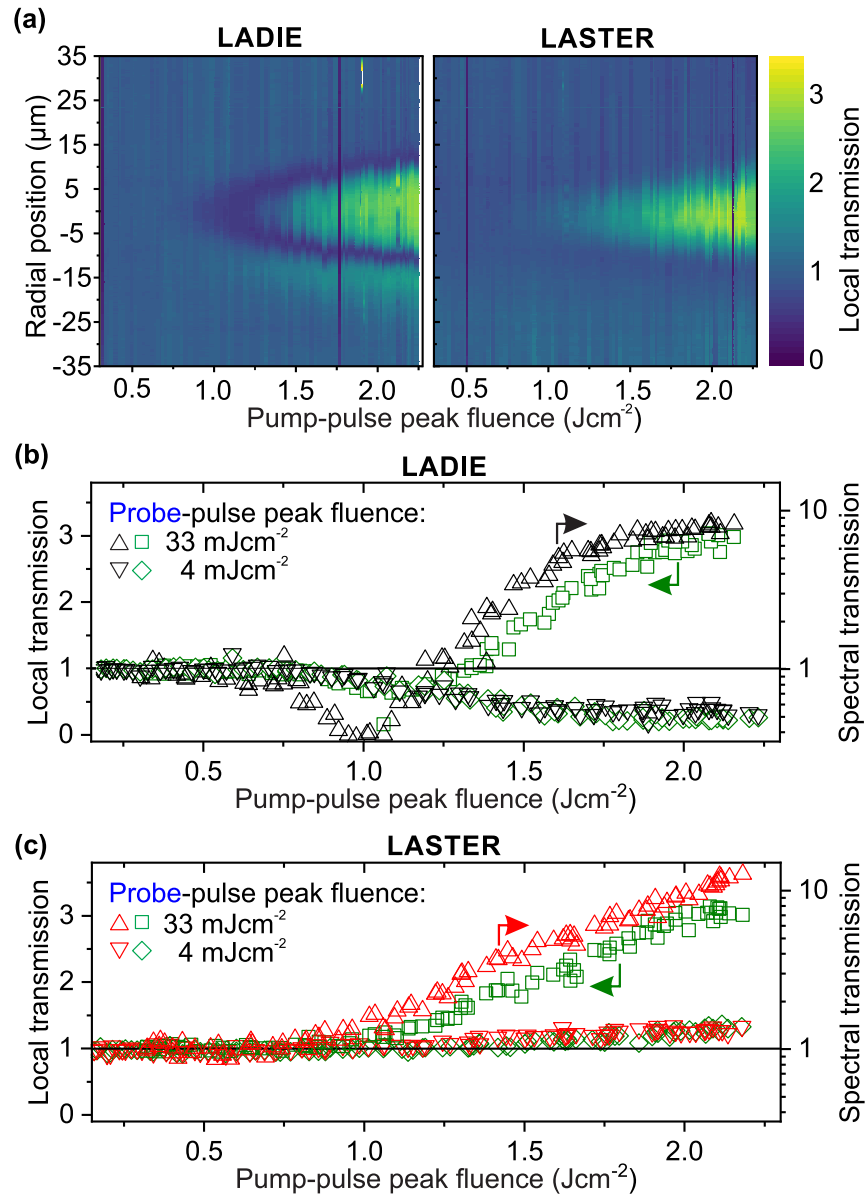

FIG. 5. Excitation dependence of LADIE and LASTER. Spatially resolved transmission as a function of the pump-pulse peak fluence (a) in the presence of conduction-band electrons (LADIE) at a 200-fs time delay and (b) in the presence of self-trapped excitons (LASTER) at $1200 \mathrm{fs}$ using a probe-pulse peak fluence of $33 \mathrm{~mJ} \mathrm{~cm}^{-2}$. Local and spectral transmission extracted from the center of the excitation (at $0 \mu \mathrm{m}$ ) under (b) LADIE and (c) LASTER conditions. Please note that the spectral transmission is shown on a logarithmic scale and that the probe-pulse peak fluences applied are indicated in the graph.

absorption and amplification follow the spatial profile of the pump pulse, causing the features seen in the center to appear at larger radii as the local pump fluence increases. We also observe a minor increase in local transmission at higher radii surrounding the absorbing regime (at lower local fluences) in Figs. 5(a) and 2(a). This observation is not made in the center of the probe pulse and can be attributed to the scattering and defocusing properties of the electron plasma, causing light to be scattered from the center towards larger radii.

For LASTER [Fig. 5(a) and green squares in Fig. 5(c)], we observe an immediate increase in local transmission at the center of the probe pulse after applying $0.85 \mathrm{~J} \mathrm{~cm}^{-2}$ peak fluence without initial absorption. This common pumpfluence threshold of transmission changes at both time delays marks the generation of free carriers by MPI, which contribute to absorption at early delay times and the later formation of STEs. As expected, with higher peak fluences, the amplification increases in the center and grows towards larger radii 
following the local pump fluence distribution. Noticeable is the lower transmission around the amplification region. This can be attributed to the focusing properties of the positiverefractive index change by the STEs, causing light from higher radii to be redistributed towards the center.

The observation of differences in the radial transmission profile, reflecting its dependence on the local pump fluence, compared to the transmission obtained from the center of excitation shows the importance of performing these spatially resolved measurements as a function of the applied pumppulse peak fluence. While the pump-fluence dependence is often extracted from the radial profile of the pump pulse as, e.g., in ultrafast reflectivity measurements [10], in transmission measurements propagation effects can alter the correct dependence [13]. By extracting the pump fluence from the center, these effects are mitigated or reduced, as the off-axis rays are much more affected by these "transient lensing" effects. While we thus do observe the influence of the refractive index changes by conduction-band electrons and STEs, respectively, it is important to note that these are independent of the amount of probe light [as shown in Figs. S2(a) and S2(b) in the Supplemental Material [36]], unlike LASTER and LADIE as we discuss in the following.

\section{Probe-pulse fluence limits: Single- vs two-photon probing}

Due to the nonlinear nature of the proposed amplification processes (LADIE and LASTER), we expect that linear onephoton effects dominate the transmission of the excited fused silica when a very low probe-pulse fluence is used. For short delay times, in the LADIE regime, this means that increasing excitation results in decreasing transmission due to the FCA $[4,10,23]$, and at late time delays, in the LASTER regime, constant transmission is expected over the given excitation range $[5,10,23]$. Because we observed that the nonlinear amplification by the excited fused silica is not present at all wavelengths [compare Figs. 4(a) and 4(b)], we distinguish the local transmission at the center of the beam and the corresponding spectral transmission of the probe pulses at $(398 \pm 0.25) \mathrm{nm}$, a wavelength close to the maximum amplification by LADIE and LASTER. As the transmission is always given as the ratio of the transmitted to the incident signal, linear, i.e., singlephoton, effects do not show a dependence of the transmission on the incident probe strength, in contrast to a two-photon signature. In Fig. 5(a), the pump-pulse fluence dependence of the local transmission under LADIE conditions ( $200 \mathrm{fs}$ ) is shown for a low (green diamonds) and a high (green squares) probe-pulse fluence. In the case of a low probe fluence, we observe a continuous decrease in both local and spectral transmission (black downward-pointing triangles) with an increase in the pump-pulse peak fluence above the excitation threshold of $0.85 \mathrm{~J} \mathrm{~cm}^{-2}$, due to FCA and the absence of nonlinear amplification. This observation is in full accordance with a manifold of experimental and theoretical investigations taking into account the dominant process of FCA via the Drude model (see [11] and references therein). This strong FCA also exists for higher probe fluences but is overcome by the growing contribution of the nonlinear amplification when increasing the probe fluence, as shown in Supplemental Fig. S3 and for excited sapphire in [3]. In comparison, the spectral transmission at $398 \mathrm{~nm}$ for a high probe-pulse fluence [Fig. 5(a); black upward-pointing triangles] shows much stronger absorption in the pump-pulse fluence range of 0.75 to $1 \mathrm{~J} \mathrm{~cm}^{-2}$, before the transmission increases up to a value of nearly 10 for a high excitation strength. We attribute this enhanced absorption to TPA, enabled by the strong bandgap renormalization, as described in our proposed model [Fig. 3(b), center] [3]. As expected, the initial absorption and following amplification are more pronounced for just the 398-nm component than for the integrated spectrum, i.e., the local transmission. Looking at the later delay time for LASTER in Fig. 5(b), the excitation dependence for local (green diamonds) and spectral (red downward-pointing triangles) transmission using a low probe fluence stays near unity for weak and strong excitation and is, thus, in accordance with what is expected from the Drude-Lorentz model $[11,14]$, where STEs only contribute with a positive refractive index change when an off-resonant probe wavelength such as $398 \mathrm{~nm}$ is used [10,11,23], measured as the positive phase shift in Fig. 2(d). Nevertheless, a small increase in local transmission can be observed for pump fluences above 1.25 $\mathrm{J} \mathrm{cm}^{-2}$, which might be related to the redistribution of light due to the focusing effect of the positive refractive index change. When a high probe fluence is used, we observe a very strong increase in spectral transmission [Fig. 5(b); red upward-pointing triangles], with values reaching and slightly exceeding one order of magnitude. Due to the missing FCA at late time delays, the local transmission (green squares) follows qualitatively the spectral transmission. To summarize, the more probe light is incident on the fused silica sample under LADIE or LASTER conditions, the higher the nonlinear amplification. This is further demonstrated in Supplemental Fig. S3 [36], which shows the local transmission as a function of the pump fluence for different probe fluences.

\section{LASTER UNDER OPTIMIZED AMPLIFICATION CONDITIONS}

We have shown in the last section that the local amplification is strongly affected if all parts of the spectrum are not amplified. This is because the local transmission carries all the spectral information. Therefore, we now discuss the amplification properties of an optimized measurement in which a probe pulse with an optimized spectrum centered around $398 \mathrm{~nm}$ was used [see Supplemental Fig. S4(a) [36]]. In Fig. 6(a), the local (green squares) and global (black circles) transmissions are shown as a function of the pump-pulse peak fluence using a probe-pulse peak fluence of $36 \mathrm{~mJ} \mathrm{~cm}^{-2}$, taken at a delay time of $1200 \mathrm{fs}$. This measurement shows that the local transmission can reach a factor of 8 , which is more than two times higher than under the conditions corresponding to Figs. 2(b) and 2(c). The global transmission rises under these optimized conditions to nearly 1.15 before it drops at higher pump-pulse fluences [compare Fig. 2(c)]. It is important to note that also under these conditions, amplification of the probe pulse has a strong influence on the spectrum. In Fig. 6(b), the FWHM spectral width of the transmitted probe pulse is shown. The width decreases by nearly a factor of 2, from approximately 8.5 to $4.5 \mathrm{~nm}$, providing a clear signature of gain narrowing, which is a typical observation 


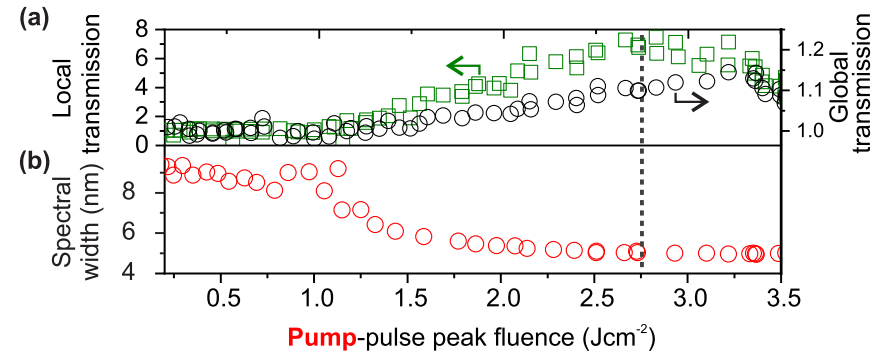

(c)

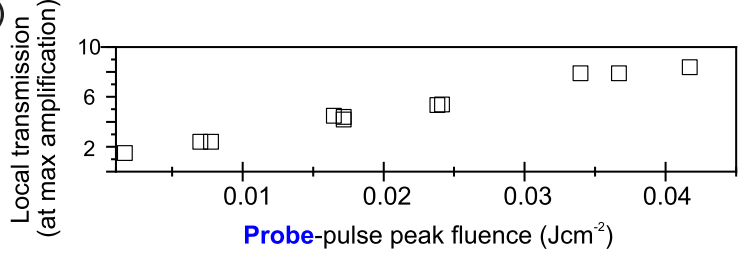

FIG. 6. Properties of the LASTER process under optimized amplification conditions. (a) Local (green squares) and global (black circles) transmission as a function of the pump-pulse peak fluence, taken at a delay time of $1200 \mathrm{fs}$. (b) Full width at half-maximum spectral width of the transmitted probe pulse as a function of the pump-pulse peak fluence. Probe-pulse peak fluence in (a) and (b) is $34 \mathrm{~mJ} \mathrm{~cm}^{-2}$. (c) Local transmission as a function of the probe-pulse peak fluence, using a pump-pulse peak fluence of $2.75 \mathrm{~J} \mathrm{~cm}^{-2}$-as indicated by the dashed vertical lines in (a) and (b).

in stimulated-emission processes. Please see Supplemental Figs. S4(a) to S4(c) for the complete spectral information.

As we have now identified the pump-pulse fluence providing maximum local amplification, we can investigate how the local transmission behaves as a function of the probe-pulse peak fluence, i.e., how the amplification changes as a function of the probe intensity. In Fig. 6(c), the local transmission coefficient is shown as a function of the probe-pulse peak fluence, ranging from less than 1 to more than $40 \mathrm{~mJ} \mathrm{~cm}$, using a pump-pulse peak fluence of $2.75 \mathrm{~J} \mathrm{~cm}^{-2}$. While this pump fluence is slightly above the ablation threshold of the material, it is important to measure the probe-fluence dependence when the amplification is strongest to be comparable to our investigation of sapphire [3]. The measurement shows a clear nonlinear behavior, as the transmission (i.e., amplification factor) increases with increasing probe strength. We also observe the onset of stagnation of the amplification factor at large probe fluences, which might be caused by the saturation of gain and/or (linear and nonlinear) reabsorption of the strong probe pulse within the sample, similar to the manifold of processes involved in the LADIE effect [3]. Therefore, only more elaborate simulations of the probe-pulse propagation through the excited fused silica and its various interaction can give deeper insight into the interaction dynamics.

\section{SUMMARY}

In summary, we utilize femtosecond pump-probe spectroscopy to demonstrate the ability to switch between two temporally separated nonlinear amplification regimes in optically excited fused silica. One amplification regime is identified as the LADIE process, which is present at times of 100 to $300 \mathrm{fs}$ after excitation. Clearly separated by an additional pump-probe delay time of $100 \mathrm{fs}$, the LASTER process is cor- related with the ultrafast formation of long-lived self-trapped excitons. We present common features and differences between the two unique nonlinear amplification regimes. While LADIE always competes with free-carrier and nonlinear absorption, the LASTER process does less so, which leads to more efficient amplification. These characteristics, together with the very long (nano- to microseconds) lifetime of population inversion over a multi-eV gap, could make LASTER a suitable candidate for realization of deep-UV or two-photon laser devices. As another possible application for both LADIE and LASTER, we imagine new types of highly nonlinear microscopy and multiphoton spectroscopy, especially in submicrometer to nanoscale localized spatial regions, as we have already demonstrated that LADIE conditions can be spatially localized in subwavelength regions by pumping with temporal Airy pulses $[3,17]$. In addition, the impact of the LASTER effect on high-repetition-rate laser material processing of fused silica and other oxide glasses, with an emphasis on optical data storage [37,38] such as Microsoft's Project Silica [39], must be investigated in future studies.

\section{ACKNOWLEDGMENTS}

We thank Lasse Haahr-Lillevang for his contributions to an earlier version of this experiment and Søren Møller for his input to discussions. We acknowledge financial support from the Deutsche Forschungsgemeinschaft (DFG), the OttoBraun-Fonds, and the Danish Council for Independent Research|Natural Sciences. This project received funding from the European Union's Horizon 2020 research and innovation program under Marie Skłodowska-Curie Grant Agreement No. 838772.

T.W., C.S., and B.Z. conceived and built the setup. T.W., N.J., and R.C. performed the measurements. Data were evaluated by T.W. The manuscript was written by T.W. All authors contributed to the discussion and proofreading of the manuscript.

\section{APPENDIX: EXPERIMENTAL SETUP}

All measurements were performed using a common-path, ultrafast-imaging (reference) pump-probe setup, which is described in detail in [3] and the associated Supplemental Material [36]. Only a short description of the fundamental aspects of the pump-probe-imaging setup is thus provided.

In short, single $800-\mathrm{nm}$ pulses with a duration of $29 \mathrm{fs}$ (FHWM) provided by an amplified Ti:sapphire laser system (Femtolasers Femtopower Pro) are split into a pump and a probe arm with a 50/50 beam splitter. The probe path is delayed with respect to the pump path using a high-precision motorized delay stage before the beam is frequency-doubled. Afterwards, the pulse is precompressed in a prism compressor stage before being focused onto a $100-\mu \mathrm{m}$-thin UV-grade and polished fused silica sample using an achromatic longworking-distance microscope objective (Mitutoyo M Plan APO $2 \times$ ). The temporal compression by the prism compressor is optimized in the interaction/sample area using a $\mathrm{SiC}$ photodiode that exhibits two-photon absorption at $400 \mathrm{~nm}$ and is therefore sensitive to the peak intensity of the pulse, i.e., its compression. Subsequently, the probe pulse is imaged onto 
the entrance slit of an imaging spectrometer by a combination of a $20 \times$ infinite-corrected, achromatic microscope objective (Mitutoyo) and a 400-mm lens, giving a total magnification of $40 \times$. The radially resolved spectrum from the spectrometer is detected by a cooled CCD array with a resolution of 128 pixel spatially and 1024 pixel spectrally (Princeton Instruments 320 spectrometer with a cooled Roper CCD array). A fraction of the probe beam is sent onto a monochromatic 1.4-Mpixel CCD camera (Lumenera LU135M) using a pellicle beam splitter to avoid ghost images from internal reflections. This CCD camera is used to resolve local and global transmission changes. Please note that at all times during the experiments, the imaged probe pulse was well centered on the camera and never exceeded an illuminated pixel area of more than $25 \%$ to $40 \%$. Optionally, a second pellicle beam splitter could be used to send a fraction of the beam onto a calibrated UV photodiode, measuring the transmitted probe-pulse energy. However, we found that these data can also be obtained by analyzing the data from the CCD camera. The incident energy of each probe pulse is measured in front of the microscope objective using a UV photodiode that receives a fraction of the probe pulse from a reflecting neutral density filter. This photodiode is calibrated against the energy recorded via a commercial power meter placed in the interaction area. A homebuilt "sample and hold" electronic enables the measurement of single pulses.

The pump-pulse path initially passes an LCD-based temporal pulse shaper [40] to precompress the pulses. Then the pump beam is recombined with the probe path using a dichroic beam splitter and focused onto the sample. Afterwards, the pump beam is blocked by two $400 \pm$ $25 \mathrm{~nm}$ OD4 interference bandpass filters. The energy of each pump pulse is measured by a fraction of the pump beam that is sent onto a calibrated photodiode (see above). The pulse duration of the pump pulse was measured by using second-order shaper-based autocorrelation [17] in the interaction area and determined to be 29-fs FWHM. The 45-fs FWHM probe-pulse duration was obtained from a low-pumpenergy cross-correlation measurement, utilizing the intensitydependent optical Kerr effect inside the fused silica sample $[13,22]$.

All experiments shown were performed in single-shot operation and between each subsequent shot the sample was moved to a new position by a high-precision motorized $x-y$ stage (PI-miCos) to ensure an undamaged area of fused silica.
[1] M. Matthews, F. Morales, A. Patas, A. Lindinger, J. Gateau, N. Berti, S. Hermelin, J. Kasparian, M. Richter, T. Bredtmann, O. Smirnova, J.-P. Wolf, and M. Ivanov, Nat. Phys. 262, 1229 (2018).

[2] G. Vampa, T. J. Hammond, D. N. Neshev, A. Y. Naumov, P. B. Corkum, and T. Brabec, Science 359, 673 (2018).

[3] T. Winkler, L. Haahr-Lillevang, C. Sarpe, B. Zielinski, N. Götte, A. Senftleben, P. Balling, and T. Baumert, Nat. Phys. 14, 74 (2018).

[4] P. Audebert, P. Daguzan, A. Dos Santos, J. C. Gauthier, J. P. Geindre, S. Guizard, G. Hamoniaux, K. Krastev, P. Martin, G. Petite, and A. Antonetti, Phys. Rev. Lett. 73, 1990 (1994).

[5] L. Haahr-Lillevang, K. Wædegaard, D. B. Sandkamm, A. Mouskeftaras, S. Guizard, and P. Balling, Appl. Phys. A 120, 1221 (2015).

[6] S. Ghimire and D. A. Reis, Nat. Phys. 15, 10 (2019).

[7] J. Siegel, D. Puerto, W. Gawelda, G. Bachelier, J. Solis, L. Ehrentraut, and J. Bonse, Appl. Phys. Lett. 91, 082902 (2007).

[8] S. Nolte, M. Will, J. Burghoff, and A. Tuennermann, Appl. Phys. A 77, 109 (2003).

[9] T. T. Fernandez, J. Siegel, J. Hoyo, B. Sotillo, P. Fernandez, and J. Solis, J. Phys. D 48, 155101 (2015).

[10] M. Garcia-Lechuga, L. Haahr-Lillevang, J. Siegel, P. Balling, S. Guizard, and J. Solis, Phys. Rev. B 95, 214114 (2017).

[11] P. Balling and J. Schou, Rep. Prog. Phys. 76, 036502 (2013).

[12] I. Mirza, N. M. Bulgakova, J. Tomáštík, V. Michálek, O. Haderka, L. Fekete, and T. Mocek, Sci. Rep. 6, 39133 (2016).

[13] T. Winkler, C. Sarpe, N. Jelzow, L. H. Lillevang, N. Götte, B. Zielinski, P. Balling, A. Senftleben, and T. Baumert, Appl. Surf. Sci. 374, 235 (2016).

[14] C. Fourment, B. Chimier, F. Deneuville, D. Descamps, F. Dorchies, G. Duchateau, M.-C. Nadeau, and S. Petit, Phys. Rev. B 98, 155110 (2018).

[15] E. G. Gamaly and A. V. Rode, Appl. Phys. A 124, 278 (2018).

[16] R. R. Gattass and E. Mazur, Nat. Photon. 2, 219 (2008).
[17] N. Götte, T. Winkler, T. Meinl, T. Kusserow, B. Zielinski, C. Sarpe, A. Senftleben, H. Hillmer, and T. Baumert, Optica 3, 389 (2016).

[18] S. Courvoisier, N. Götte, B. Zielinski, T. Winkler, C. Sarpe, A. Senftleben, L. Bonacina, J. P. Wolf, and T. Baumert, APL Photon. 1, 046102 (2016).

[19] G. Campargue, B. Zielinski, S. Courvoisier, C. Sarpe, T Winkler, L. Bonacina, T. Baumert, and J. P. Wolf, AIP Adv. 8, 125105 (2018).

[20] Y. R. Shen, Phys. Lett. 20, 378 (1966).

[21] M. N. Islam, J. R. Simpson, H. T. Shang, L. F. Mollenauer, and R. H. Stolen, Opt. Lett. 12, 625 (1987).

[22] C. Sarpe, J. Köhler, T. Winkler, M. Wollenhaupt, and T. Baumert, New J. Phys. 14, 075021 (2012).

[23] S. S. Mao, F. Quéré, S. Guizard, X. Mao, R. E. Russo, G. Petite, and P. Martin, Appl. Phys. A 79, 1695 (2004).

[24] N. M. Bulgakova, R. Stoian, and A. Rosenfeld, Quantum Electron. 40, 966 (2010).

[25] L. V. Keldysh, Sov. Phys. JETP 20, 1307 (1965).

[26] K. Huthmacher, A. K. Molberg, B. Rethfeld, and J. R. Gulley, J. Comput. Phys. 322, 535 (2016).

[27] D. K. Ferry, Phys. Rev. B 18, 7033 (1978).

[28] S. V. Faleev, M. van Schilfgaarde, T. Kotani, F. Léonard, and M. P. Desjarlais, Phys. Rev. B 74, 033101 (2006).

[29] K. Sokolowski-Tinten and D. von der Linde, Phys. Rev. B 61, 2643 (2000).

[30] M. Schultze, K. Ramasesha, C. D. Pemmeraju, S. A. Sato, D. Whitmore, A. Gandman, J. S. Prell, L. J. Borja, D. Prendergast, K. Yabana, D. M. Neumark, and S. R. Leone, Science 346, 1348 (2014).

[31] S. Pagliara, G. Galimberti, S. Mor, M. Montagnese, G. Ferrini, M. S. Grandi, P. Galinetto, and F. Parmigiani, J. Am. Chem. Soc. 133, 6318 (2011).

[32] T. Nagai, T. J. Inagaki, and Y. Kanemitsu, Appl. Phys. Lett. 84, 1284 (2004). 
[33] B. Rethfeld, Phys. Rev. Lett. 92, 187401 (2004).

[34] B. H. Christensen and P. Balling, Phys. Rev. B 79, 155424 (2009).

[35] Y. D. Glinka, S.-H. Lin, L.-P. Hwang, Y.-T. Chen, and N. H. Tolk, Phys. Rev. B 64, 085421 (2001).

[36] See Supplemental Material at http://link.aps.org/supplemental/ 10.1103/PhysRevResearch.2.023341 for supplemental figures on pump- and probe-fluence dependencies as well as spectral information of the LASTER amplification under optimized conditions.

[37] J. Zhang, M. Gecevicius, M. Beresna, and P. G. Kazansky, Phys. Rev. Lett. 112, 033901 (2014).
[38] A. Okhrimchuk, S. Fedotov, I. Glebov, V. Sigaev, and P. Kazansky, Sci. Rep. 7, 16563 (2017).

[39] P. Anderson, R. Black, A. Cerkauskaite, A. Chatzieleftheriou, J. Clegg, C. Dainty, R. Diaconu, A. Donnelly, R. Drevinskas, A. Gaunt, A. Georgiou, A. G. Diaz, P. G. Kazansky, D. Lara, S. Legtchenko, S. Nowozin, A. Ogus, D. Phillips, A. Rowstron, M. Sakakura, I. Stefanovici, B. Thomsen, and L. Wang, in 10th USENIX Workshop on Hot Topics in Storage and File Systems (HotStorage 18) (USENIX Association, Berkeley, CA, 2018), pp. 1-6.

[40] J. Köhler, M. Wollenhaupt, T. Bayer, C. Sarpe, and T. Baumert, Opt. Express 19, 11638 (2011) 